\title{
CLASSICAL FEATURES OF THE MOTION OF A HEAVY BEAD SLIDING ON A ROTATING WIRE
}

\author{
Ata Abu-As' AD $^{1}$, Hussein ShanaK ${ }^{2}$, Jihad AsaD $^{2 *}$ \\ ${ }^{1}$ Dept. of Applied Mathematics, College of Science, Palestine Technical \\ University, P.O. Box 7, Tulkarm, Palestine \\ ${ }^{2}$ Dept. of Physics, College of Science, Palestine Technical University, P.O. \\ Box 7, Tulkarm, Palestine
}

[Received: 20 January 2019. Accepted: 08 July 2019]

doi: 10.7546/JTAM.49.19.03.02

\begin{abstract}
In this paper, we study the motion of a heavy bead sliding on a rotating wire. Our first step was constructing the classical Lagrangian of the system. Secondly, we derived the Euler- Lagrange equation (ELE). Thirdly, we solve the obtained ELE, which is a non-homogenous second order linear differential equation. Finally, by using MATLAB the equation is solved numerically for some selected parameters, and for specified initial conditions.
\end{abstract}

KEY WORDS: Heavy particle, Lagrange equation, Euler-Lagrange equation, Particular solution, Homogenous solution, Numerical solution.

\section{INTRODUCTION}

Lagrangian and Hamiltonian mechanics play an important role in solving a wide range of classical physical systems [1-3]. This branch of classical mechanics is based on scalars concepts (i.e. kinetic and potential energies). Classical mechanics books contain many such systems, and for more details one can refer to the three references [1-3] above. Solving such systems by this technique results in obtaining differential equations called equations of motions (i.e. Euler-Lagrange equations). These equations have to be solved for some given initial conditions either analytically or numerically in some cases.

In under graduate level mathematician and physician students study an interesting course called ordinary differential equations (ODE). In this course students study techniques that enable them to solve many branches of ODE, see for example [4-7].

Numerical solution of ODE's are powerful because they help scientists in solving many kinds of DE's without the need of knowing their analytical solutions due to difficulty, or insufficient data. In literature one can find many numerical methods and techniques that has been considered [8-12].

\footnotetext{
${ }^{*}$ Corresponding author e-mail: drjasad@yahoo.com
} 
In this paper, we choose an interesting physical system (a heavy bead sliding in a rotating wire). The importance of this example is due to the fact that the kinetic energy of it depends on both the dynamical variable and its derivative, instead on the time derivative only as it is familiar [3]. In addition, problems similar to the system considered in this work arise when we study dynamics of mechanical systems with rotating parts performing various operations such as mixing, grinding, drying, etc. of diverse substances [13-15]. In our system we considered the simplest situation where friction is neglected.

The rest of this work is organized as follows: In Section 2 an explanation how to obtain the Euler-Lagrange equation of the system is illustrated. Analytical and numerical solution for the system is given in Section 3. Results and discussion are presented in Sec. 4. Finally, we close the paper with a conclusion.

\section{Physical Description of the System and its Euler-Lagrange EQUATIONS}

Consider a heavy bead of mass $(m)$ sliding without friction on a thin wire that is rotating about a vertical axis by an external agent (i.e. motor) at a constant angular frequency $(\omega)$ as illustrated in Fig. 1 below.

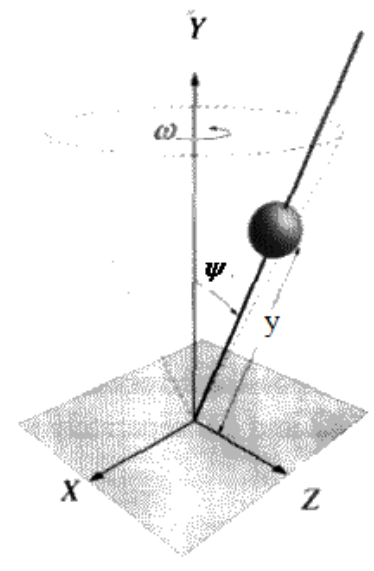

Fig. 1. Heavy bead sliding on a rotating wire.

The wire is tilted away from the vertical $y$-axis by a fixed angle $\psi$. The bead is constrained to move on the wire. The importance of this example comes from the fact that the kinetic energy in this case depends on both the dynamical variable and on its derivative, instead of on the time derivative alone.

To write the classical Lagrangian $(L)$ of the system we need to write both the kinetic energy and the potential energy of the bead respectively, then, as it is known 
$L=T-V$. To describe the motion of the bug we need just one dynamical variable which is $q$ (i.e., the distance from origin). Thus (for more details about the derivation one can refer to [3])

$$
L=T-V=\frac{1}{2} m\left(\dot{y}^{2}+y^{2} \omega^{2} \sin ^{2} \psi\right)-m g y \cos \psi .
$$

Now, by using the known equation

$$
\frac{\partial L}{\partial y}-\frac{d}{d t} \frac{\partial L}{\partial \dot{y}}=0
$$

the classical Euler-Lagrange equation read

$$
\ddot{y}=y \omega^{2} \sin ^{2} \psi-g \cos \psi .
$$

The last equation can now be solved for specific parameters if some initial conditions are given.

\section{Analytical and Numerical Solution of the System}

In this section we aim to solve Eq. (2) analytically and numerically for some specified initial conditions. First of all let us introduce the following two variables $\alpha$ and $\beta$, where:

$$
\begin{aligned}
& \alpha=\omega \sin \psi, \\
& \beta=g \cos \psi .
\end{aligned}
$$

Using Eqs. (3) and (4), Eq. (2) becomes

$$
\ddot{y}-\alpha^{2} y=-\beta .
$$

Following the procedures known in the ordinary differential equations texts for undergraduate levels (see, for example, [5,6]) one can easily solve Eq. (5) above easily. From Literature it is clear that the solution of Eq. (5) consists from two parts of homogenous solution $\left(y_{h}\right)$, and the particular solution $\left(y_{p}\right)$. The first one can be obtained by setting the right hand side of Eq. (5) to zero, then the following homogenous solution is obtained (for more details the reader can refer to [2-5]):

$$
y_{h}(t)=A \mathrm{e}^{\alpha t}+B \mathrm{e}^{-\alpha t} .
$$

Concerning the particular solution and since the right hand side of Eq. (5) is constant, we can assume the particular solution as

$$
y_{p}(t)=C \text {. }
$$


After substituting Eq. (7) in Eq. (5), one gets

$$
y_{p}=\frac{g \cos \psi}{\omega^{2} \sin ^{2} \psi} .
$$

As a result, the general solution to Eq. (5) is the sum of Eq. (7) and Eq. (8). Therefore, the general solution can be written as

$$
y(t)=A \mathrm{e}^{(\omega \sin \psi) t}+B \mathrm{e}^{-(\omega \sin \psi) t}+\frac{g \cos \psi}{\omega^{2} \sin ^{2} \psi} .
$$

Making use of the following initial condition: $y(0)=5 \mathrm{~m} \dot{y}(0)=0$, one can write Eq. (9) as

$$
y(t)=\left(5-\frac{g \cos \psi}{\omega^{2} \sin ^{2} \psi}\right) \cosh ((\omega \sin \psi) t)+\frac{g \cos \psi}{\omega^{2} \sin ^{2} \psi} .
$$

Below we make use of MATLAB in solving Eq. (10) numerically for some selected parameters and the initial condition given above as presented in Figs. 2 - 4.

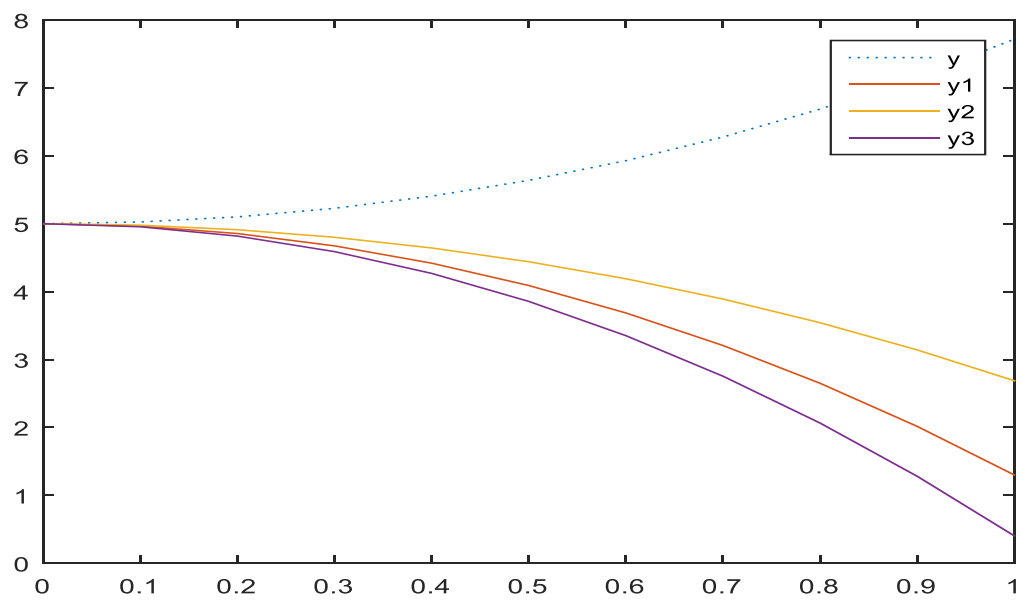

Fig. 2. (Color online) The variation of the distance against time, $\omega=1, \psi=$ $\pi / 2, \pi / 6, \pi / 4, \pi / 12, t=0.1 i, i=0,1, \ldots, 10$, where $y, y_{1}, y_{2}$ are respect to $\psi$.

Now, if we consider another new initial condition $y(0)=5, \dot{y}(0)=1$, the general solution of Eq. (9) will be

$$
y(t)=\left[5-\left(\frac{1}{\omega \sin \psi}+\frac{g \cos \psi}{\omega^{2} \sin ^{2} \psi}\right)\right] \cosh ((\omega \sin \psi) t) .
$$

Again, we make use of MATLAB in solving Eq. (11) numerically for some selected parameters and the initial condition given above as presented in Figs. 5 - 9 . 
228 Classical Features of the Motion of a Heavy Bead Sliding on a Rotating Wire

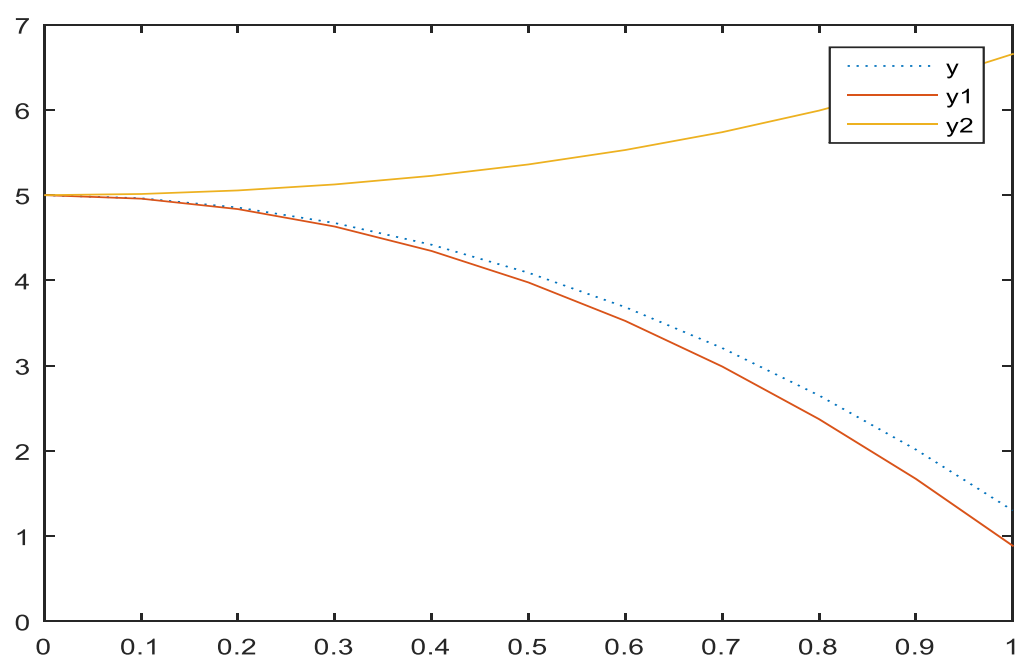

Fig. 3. (Color online) The variation of the distance against time, $\omega=(0.5,1,3), \psi=\pi / 6$, $t=0.1 i, i=0,1, \ldots, 10$, where $y, y_{1}, y_{2}$ are respect to $\omega$.

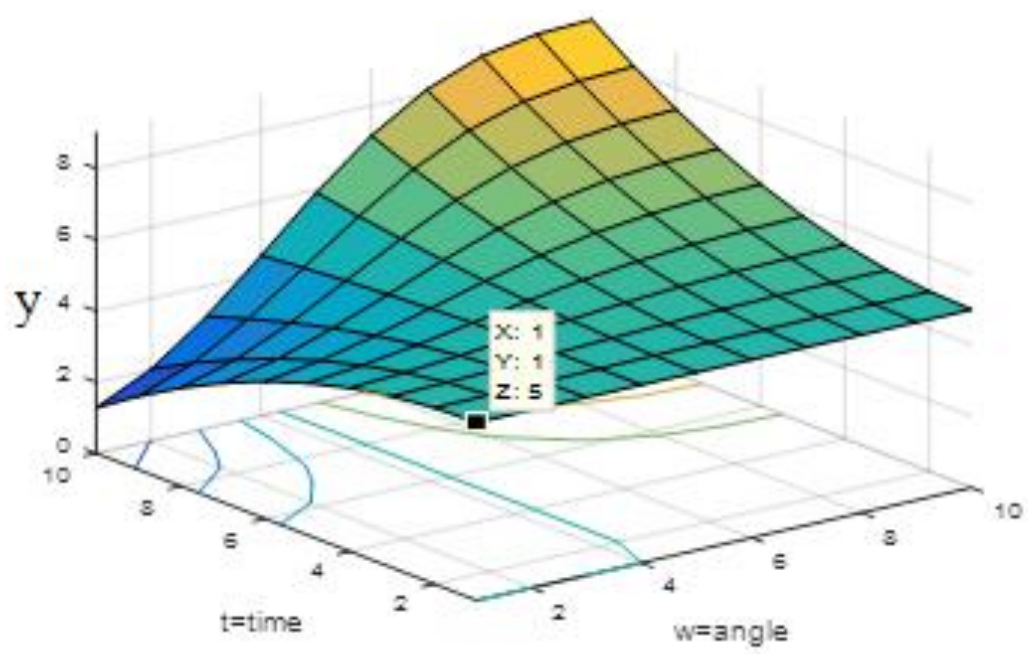

Fig. 4. (Color online) The variation of the distance against time, and the angle $\psi$ for fixed angular speed $\omega=1$. Here $\psi=(\pi / 12) i, t=0.1 i, i=0,1, \ldots, 10$.

\section{RESUlts AND Discussion}

Figures $2-9$ show the position $y(t)$ of the particle against the time. In Figs. 2 4 we use the initial conditions $y(0)=5, \dot{y}(0)=0$ to obtain the constant $A$ and $B$ 


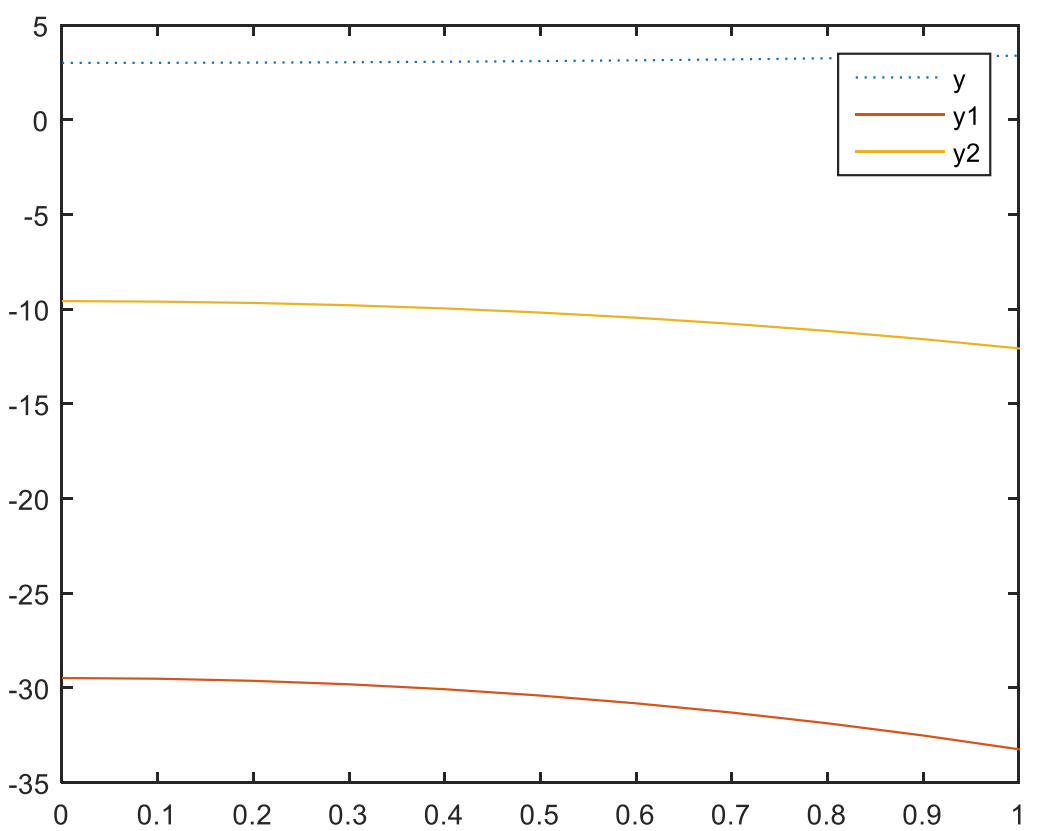

Fig. 5. (Color online) The variation of the distance against time for $\omega=0.5$ and the following angles $\psi=(\pi / 2, \pi / 6, \pi / 4)$, where $y, y_{1}, y_{2}$ belong to $\psi$, respectively.

given in Eq. (9) and then we plot $y(t)$ against time. Figure 2 shows the variation of position $y(t)$ against time when angular speed is fixed and chosen to be $\omega=1$, while the angle $\psi$ is changing (here we consider $\psi=\pi / 2, \pi / 6, \pi / 4, \pi / 12$ ). On the other hand in Fig. 3 the variation of the position $y(t)$ against time is plotted when the angular speed is fixed and chosen to be $\omega=3$, while the angle $\psi$ is changing (here we consider $\psi=\pi / 2, \pi / 6, \pi / 4, \pi / 12)$. Figure 4 shows the behavior of $y(t)$ against both time and the angle $\psi$ for constant angular speed $\omega=1$.

In Figs. $5-7$ we show the variation of the distance against time for different angles $\psi=\pi / 2, \pi / 6, \pi / 4$, where in Fig. 5 angular speed is taken $\omega=0.5$, in Fig. 6 $\omega=1$, and finally in Fig. $7 \omega=3$. In the last two figures (Figs. 8 and 9) the variation of the distance against tine for different angular speeds $\omega=0.5,1,3$, in Fig. 8 the angle is chosen to be $\psi=\pi / 4$, while in Fig. $9 \psi=\pi / 12$.

In Fig. 2 one can see that for $\psi=\pi / 2$ the distance is increasing (the particle is moving away from the origin (i.e. moving to the right) as it is expected because the wire is rotating in a horizontal plane, and it is not inclined. But for the angles $\psi=\pi / 6, \pi / 4, \pi / 12$ the distance is decreasing. This is due to the fact that the bead is sliding towards the origin and it is sliding faster for $\psi=\pi / 12$ rather than $\psi=\pi / 6$, $\pi / 4$. 
230 Classical Features of the Motion of a Heavy Bead Sliding on a Rotating Wire

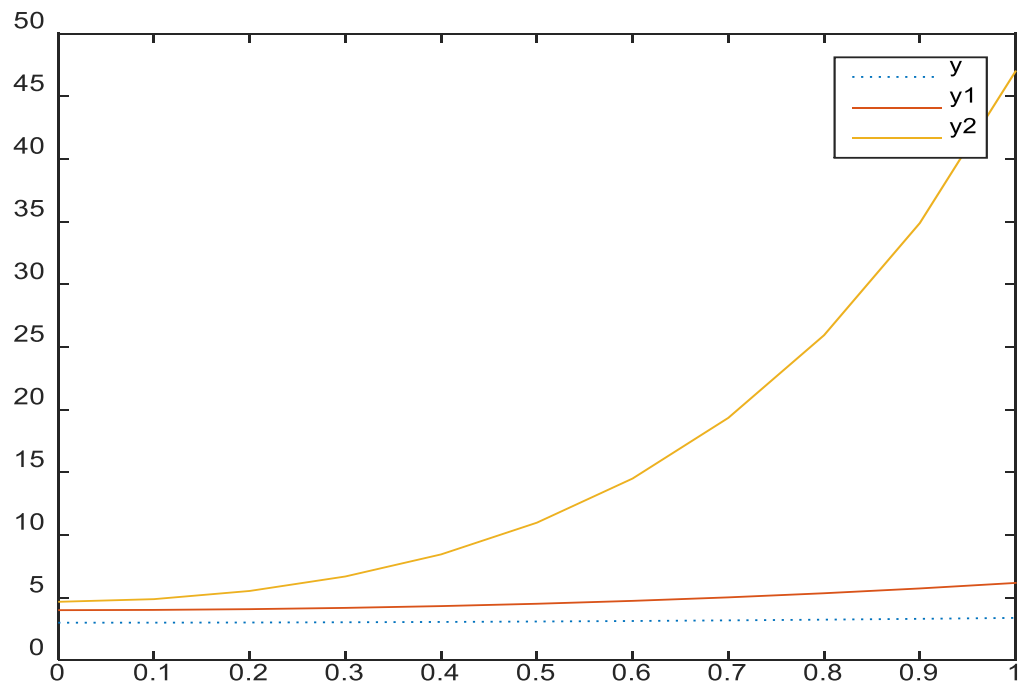

Fig. 6. (Color online) The variation of the distance against time for $\omega=1$ and the following angles $\psi=(\pi / 2, \pi / 6, \pi / 4)$, where $y, y_{1}, y_{2}$ belong to $\psi$, respectively.

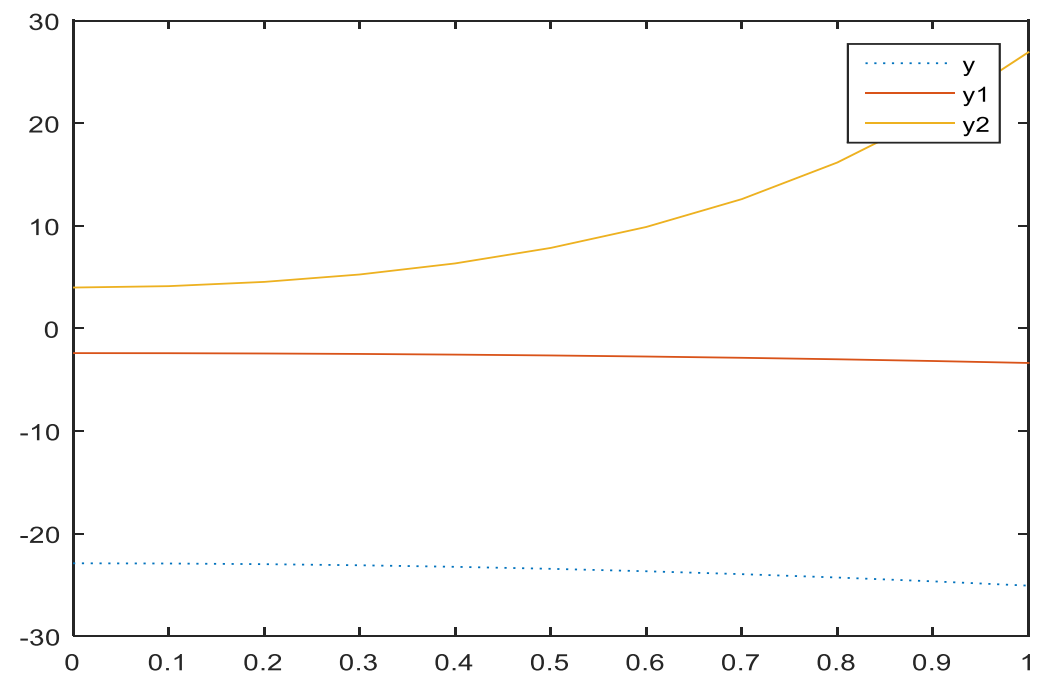

Fig. 7. (Color online) The variation of the distance against time for $\omega=3$ and the following angles $\psi=(\pi / 2, \pi / 6, \pi / 4)$, where $y, y_{1}, y_{2}$ belong to $\psi$, respectively. 


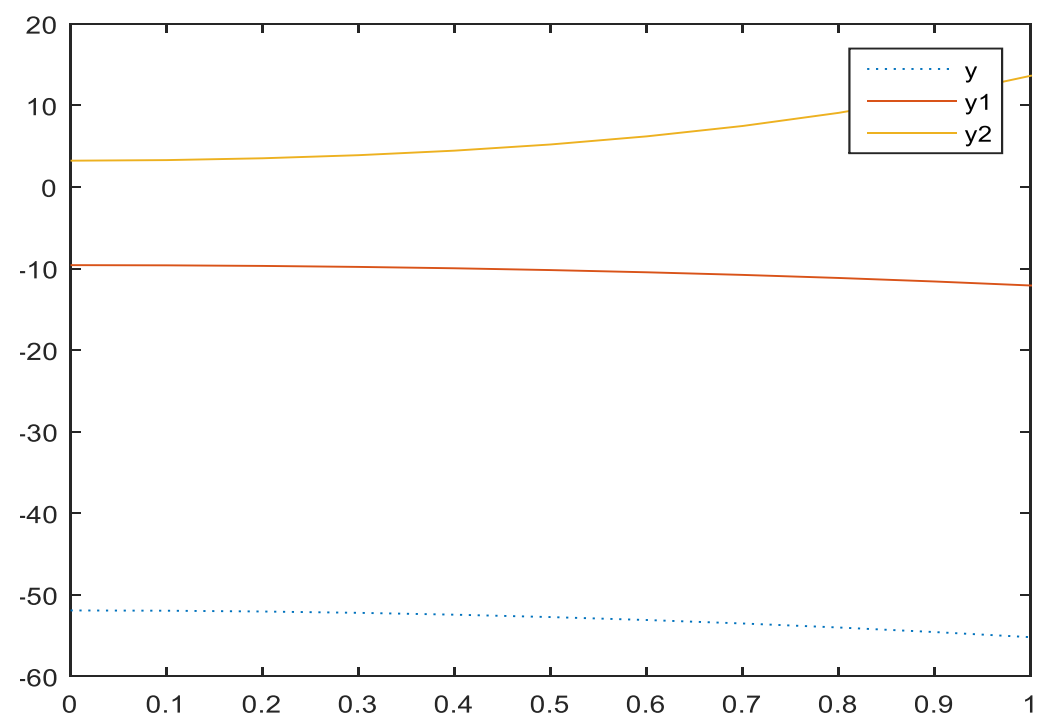

Fig. 8. (Color online) The variation of the distance against time for the angle $\psi=\pi / 4$ and the following angular speeds $\omega=0.5,1,3$, where $y, y_{1}, y_{2}$ belong to $\omega$, respectively.

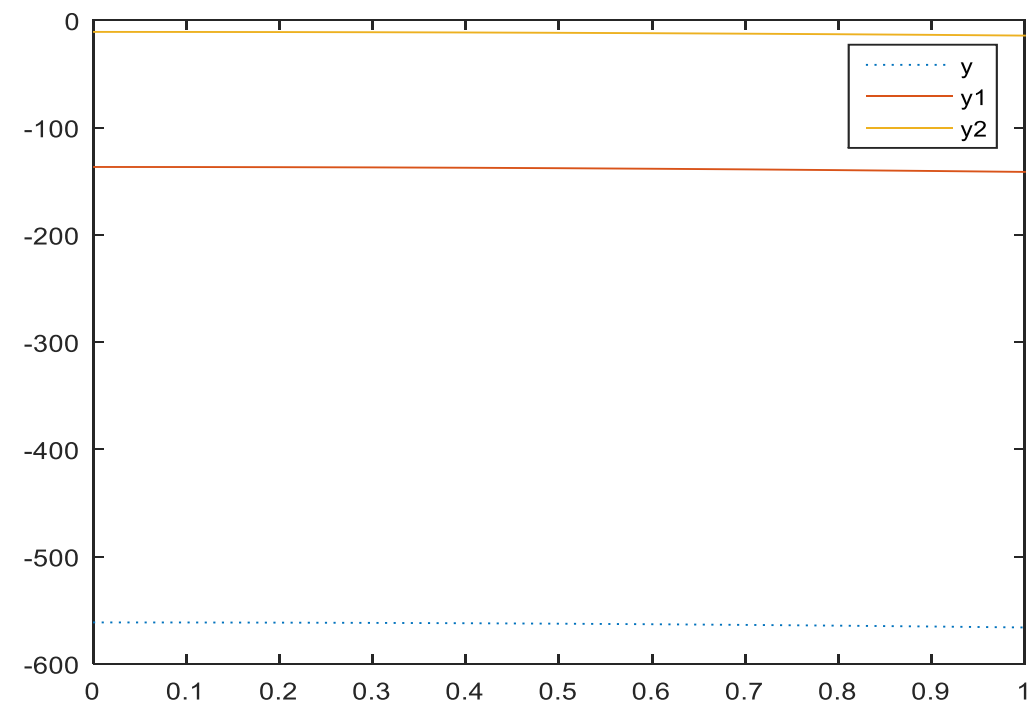

Fig. 9. (Color online) The variation of the distance against time for the angle $\psi=\pi / 12$ and the following angular speeds $\omega=0.5,1,3$, where $y, y_{1}, y_{2}$ belong to $\omega$, respectively. 
In Fig. 3 it is clear that increasing $\omega$ results in faster sliding towards the origin. Figure 5 shows that since the angular speed is small (i.e. $\omega=0.5$ ) the variation in distance against time is not clear. In Figs. 6 and 7, when $\omega=1$, the variation in distance against time is clearer than that when $\omega=3$.

\section{REFERENCES}

[1] G.R. Fowles, G.L. CASsiday (2005) "Analytical Mechanics", 7th ed., Thomson Brooks/Cole.

[2] J.B. Marion, S.T. Thornton (1988) "Classical Dynamics of Particles and Systems", 3rd ed. Harcourt Brace Jovanovich.

[3] L.N. HAND, J.D. Finch (1988) "Analytical Mechanics". Cambridge University Press.

[4] V.I. ARnold (1989) "Mathematical Methods of Classical Mechanics", 2nd ed. Springer-Verlag, New York Inc.

[5] J.L. BRenner (2013) "Problems in Differential Equations". Dover Publication. Mineola. New York.

[6] V.I. ARnold (1989) "Ordinary Differential Equations", 1st ed. The MIT Press, Cambridge, England.

[7] S.P. Mondal, T.K. Roy (2013) First Order Linear Non Homogeneous Ordinary Differential Equation in Fuzzy Environment. Mathematical Theory and Modeling 3(1) 8595.

[8] W.C. Rheinboldt (1995) Performance Analysis of Some Methods for Solving EulerLagrange Equations. Applied Mathematics Letters 8(1) 77-82.

[9] F.A. Porta, J. YEn (2007) Implicit Numerical Integration for Euler-Lagrange Equations via Tangent Space Parameterization. Mechanics of Structures and Machines 19 77-98.

[10] K. Atkinson, W. Han, D. Stewart (2008) "Numerical Solution of Ordinary Differential Equations". Wiley.

[11] H. Khalilia, R. Jarrar, J. ASAD (2018) Numerical Study of Motion of a Spherical Particle in a Rotating Parabola Using Lagrangian. Journal of the Serbian Society for Computational Mechanics. 12(1) 44-45.

[12] J.C. ButcheR (2008) "Numerical Methods for Ordinary Differential Equations", 2nd ed. Wiley.

[13] N. Van De Wouw, M. Van Den Heuvel, H. Nijmeijer, J. Van Rooij (2005) Performance of an automatic ball balancer with dry friction. International Journal of Bifurcation and Chaos 15(1) 65-82.

[14] P. Joshi, K.D.P. Nigam, E.B. Nauman (1995) The Kenics Static Mixer: New Data and Proposed Correlations. Chemical Engineering Journa 59(3) 265-271.

[15] A. Burov, E. Shalimova (2017) Bifurcations of Relative Equilibria Sets of a Massive Point on Rough Rotating Surfaces. Technische Mechanik 37(2-5) 129-138. 